\title{
Crystal fields in Sc, Y, and the heavy-rare-earth metals Tb, Dy, Ho, Er, Tm, and Lu
}

\section{Touborg, P.}

\section{Published in:}

Physical Review B

Link to article, DOI:

10.1103/PhysRevB.16.1201

Publication date:

1977

\section{Document Version}

Publisher's PDF, also known as Version of record

Link back to DTU Orbit

Citation (APA):

Touborg, P. (1977). Crystal fields in Sc, Y, and the heavy-rare-earth metals Tb, Dy, Ho, Er, Tm, and Lu. Physical Review B, 16(3), 1201-1211. https://doi.org/10.1103/PhysRevB.16.1201

\section{General rights}

Copyright and moral rights for the publications made accessible in the public portal are retained by the authors and/or other copyright owners and it is a condition of accessing publications that users recognise and abide by the legal requirements associated with these rights.

- Users may download and print one copy of any publication from the public portal for the purpose of private study or research.

- You may not further distribute the material or use it for any profit-making activity or commercial gain

- You may freely distribute the URL identifying the publication in the public portal

If you believe that this document breaches copyright please contact us providing details, and we will remove access to the work immediately and investigate your claim. 


\title{
Crystal fields in Sc, Y, and the heavy-rare-earth metals Tb, Dy, Ho, Er, Tm, and Lu
}

\author{
P. Touborg \\ Department of Electrophysics, Technical University, Lyngby, Denmark \\ and Physics Department, * University of Odense, 5000 Odense, Denmark
}

(Received 2 August 1976)

\begin{abstract}
Experimental investigations of the magnetic properties of dilute alloys of the rare-earth solutes $\mathrm{Tb}, \mathrm{Dy}$, $\mathrm{Ho}, \mathrm{Er}$, and $\mathrm{Tm}$ in the nonmagnetic hosts $\mathrm{Lu}, \mathrm{Y}$, and $\mathrm{Sc}$ have been performed. These measurements, which include and supplement earlier published results, have been analyzed and crystal-field parameters for all these 15 alloy systems deduced. The consistency of the parameters was confirmed by a variety of magnetic measurements, including neutron spectroscopy. Crystal-field parameters have also been derived for the ions in pure magnetic rare-earth metals and their alloys using the results for the dilute alloys supplemented with paramagnetic measurements up to high temperatures on the concentrated systems. Mean values and standard deviations of the higher-order crystal-field parameters for all $\mathrm{Y}$ and $\mathrm{Lu}$ alloys are $B_{40} / \beta=6.8 \pm 0.9 \mathrm{~K}$, $B_{60} / \gamma=13.6 \pm 0.7 \mathrm{~K}$, and $B_{66} / \gamma=(9.7 \pm 1.1) B_{60} / \gamma$. These values-with the inaccuracies somewhat increased-are expected to be representative also for the magnetic rare-earth metals. For rare-earth ions in the Sc host the values $B_{40} / \beta=9.9 \pm 1.9 \mathrm{~K}, B_{60} / \gamma^{\prime}=19.8 \pm 1.5 \mathrm{~K}$, and $B_{66} / \gamma=(9.4 \pm 0.9) B_{60} / \gamma$ were deduced. $B_{20} / \alpha$ is a host-sensitive parameter which has the average values of $-102.7,-53.4$, and $29.5 \mathrm{~K}$ for rare-earth ions in $\mathrm{Y}, \mathrm{Lu}$, and Sc, respectively. There is also evidence that this parameter varies with the solute. $B_{20} / \alpha$ for ions in the pure magnetic rare-earth metals and their alloys shows a linear variation with $c / a$ ratio characteristic of each ion. The results indicate a contribution from anisotropic exchange to the high-temperature paramagnetic anisotropy of approximately $20 \%$ for $\mathrm{Tb}, \mathrm{Dy}, \mathrm{Ho}$, and $\mathrm{Er}$, and approximately $10 \%$ for $\mathrm{Tm}$.
\end{abstract}

\section{INTRODUCTION}

Crystal-field parameters for a number of dilute alloys of heavy rare earths in the nonmagnetic hcp rare-earth-like metals $\mathrm{Y}, \mathrm{Lu}$, and Sc have been published earlier. ${ }^{1-5}$ The parameters were deduced by fitting theoretical expressions for the paramagnetic susceptibilities $t$, the experimental results at low fields. As demonstrated for $\mathrm{Y}-\mathrm{Dy}$ by neutron spectroscopy, ${ }^{6}$ errors in some of the published parameters may exist due to the effects of magnetic ordering on the susceptibility in the hard direction at low temperatures. The correct paramagnetic susceptibility can however be obtained from isothermal magnetization curves as the slope of the linear range following the initial high slope range, as discussed and demonstrated in Ref.6. In the present work the earlier published measurements of the initial susceptibility have been corrected. Furthermore similar measurements and corrections have been performed on the remaining binary alloys of $\mathrm{Tb}, \mathrm{Dy}, \mathrm{Ho}, \mathrm{Er}$, and $\mathrm{Tm}$ in $\mathrm{Y}, \mathrm{Lu}$, and Sc. The paramagnetic susceptibilities of these 15 alloy systems have been analyzed, and the crystal-field and molecular-field parameters for each alloy deduced. This complete set of data offers the possibility of a study of variations of parameters with host and with rareearth solute. The parameters deduced for each alloy and certain common parameters, characteristic of each host, were compared to a variety of earlier published measurements. ${ }^{2-8}$ These include magnetization measurements in different crystallographic directions over a wide range of temperatures and fields, experiments on the basal-plane magnetic anisotropy and neutron spectroscopy.

To obtain the crystal-field parameters also in the magnetic rare-earth metals $\mathrm{Tb}$ to $\mathrm{Tm}$, measurements up to high temperature of the paramagnetic susceptibilities were performed on these metals. The results of these measurements combined with the results of the study of the dilute alloys allow a reliable estimate of all crystal-field parameters in the heavy-rare-earth metals and in alloys between them. The results were compared to paramagnetic measurements on the $\mathrm{Tb}-\mathrm{Ho}$, $\mathrm{Tb}-\mathrm{Er}$, and Dy-Ho alloy systems.

\section{EXPERIMENTAL TECHNIQUES}

Single crystals were prepared using the recrystallization method described in Ref. 9. Samples for magnetization measurements were 2-4$\mathrm{mm}$ spheres formed by spark cutting. (A suitable method for preparing samples with almost ideal spherical shape is described in Ref. 10.) The samples were oriented using $\mathrm{x}$-ray Laue techniques.

The initial susceptibilities of the dilute alloys were measured using the Faraday method, for which a sensitivity sufficient for measuring these small magnetic moments could be obtained. The Faraday magnetometer ${ }^{2}$ used for the early measurements had superconducting coils for producing the main and gradient fields. Later measure- 
ments were performed on a similar Faraday magnetometer but with copper coils embedded in liquid nitrogen for producing the fields $\left(B_{\max }\right.$ $=1.5 \mathrm{~T}$ ). The strict proportionality between the fields and the currents through the copper coils is convenient for the initial susceptibility measurements.

Magnetization measurements up to $6 \mathrm{~T}$ were performed on a $5-\mathrm{Hz}$ vibrating-sample magnetometer ${ }^{2,11}$ and magnetization measurements up to $37 \mathrm{~T}$ on the University of Amsterdam high-field magnetometer. ${ }^{12}$

A $169-\mathrm{Hz}$ vibrating-sample anisotropy meter ${ }^{13}$ was used for sensitive measurements of the basal plane anisotropy. In this instrument the sample was rotated around the vertical $c$ axis, and the horizontal magnetic moment $M_{\perp}$ perpendicular to the horizontal magnetic field was measured as a function of crystal rotation angle $\phi$. The sixthorder Fourier component $M_{1,6}$ of $M_{\perp}(\phi)$ was compared to theoretical values.

The intensity spectrum of the energy of scattered neutrons with fixed wave-vector transfer and fixed incident energy was measured on a triple-axis spectrometer $^{14}$ at Ris $\phi$.
Measurements up to $700 \mathrm{~K}$ of the paramagnetic susceptibility of concentrated rare-earth metals and alloys were made with a Foner-type vibratingsample magnetometer ${ }^{15,16}$ mounted in an electromagnet. Measurements along all directions in a crystal plane could be performed by a simple rotation of the sample holder. An anisotropy of less than $0.2 \%$ in this plane could be detected.

\section{THEORY}

The Hamiltonian appropriate for interpretation of these experiments consists of crystal-field, Zeeman, and exchange terms

$$
\mathfrak{H}=\mathfrak{H}_{\mathrm{CF}}+\mathfrak{H}_{Z}+\mathfrak{H}_{\text {ex }} .
$$

The crystal-field Hamiltonian per magnetic ion is written as the one-particle potential

$$
\mathfrak{H}_{\mathrm{CF}}=\sum_{i} V_{\mathrm{CF}}\left(\overrightarrow{\mathrm{r}}_{i}\right) \text {. }
$$

The sum runs over all electrons in the ion. For rare-earth ions (in their ground configuration) in the hcp structure only the following terms contribute to the crystal-field potential:

$$
\begin{aligned}
V_{C F}(\bar{r})= & (2 \pi)^{1 / 2}\left(\frac{8}{5}\right)^{1 / 2} V_{20}(r) Y_{2}^{0}(\theta, \Phi)+(2 \pi)^{1 / 2}(16 / 3 \sqrt{2}) V_{40}(r) Y_{4}^{0}(\theta, \phi) \\
& +(2 \pi)^{1 / 2} 32 /(\sqrt{26})^{1 / 2} V_{80}(r) Y_{6}^{0}(\theta, \phi)+(2 \pi)^{1 / 2}\left[32 /(6006)^{1 / 2}\right] V_{66}(r)\left[Y_{6}^{6}(\theta, \phi)+Y_{6}^{-6}(\theta, \phi)\right] .
\end{aligned}
$$

The coordinate system is chosen with the $x, y$, and $z$ axes along the crystallographic $b, a$, and $c$ axes, respectively.

When the charges giving rise to the crystal field are entirely outside the $4 f$ ion

$$
V_{l m}(r)=A_{l m} r^{l}
$$

For the heavy-rare-earth ions, in the temperature ranges studied, contributions from the higher multiplets in the $L S$ ground-state term were found to be negligible. Within the basis $\left|J, M_{J}\right\rangle$

$$
\mathfrak{H}_{\mathrm{CF}}=B_{20} O_{2}^{\circ}(\bar{J})+B_{40} O_{4}^{\circ}(\bar{J})+B_{60} O_{6}^{\circ}(\bar{J})+B_{66} O_{6}^{6}(\bar{J}),
$$

where $O_{l}^{m}(\bar{J})$ are the Stevens operators. ${ }^{17}$ The connection to (3) is given by

$$
\left\langle V_{l m}(r)\right\rangle_{4 f}=\int\left|R_{4 f}(r)\right|^{2} V_{l m}(r) r^{2} d r=\frac{B_{l m}}{\alpha_{l}} .
$$

$\alpha_{2}=\alpha, \alpha_{4}=\beta$, and $\alpha_{6}=\gamma$ are reduced matrix elements (the Stevens factors ${ }^{17}$ ).

The Zeeman Hamiltonian is

$$
\mathfrak{H}_{Z}=g_{J} \mu_{B} \overrightarrow{\mathrm{J}} \cdot \overrightarrow{\mathrm{H}} \text {. }
$$

The exchange interaction is treated in the molecular field approximation. For the dilute alloys the exchange interaction is written

$$
\mathfrak{H}_{\text {ex }}=g_{J} \mu_{B} \overrightarrow{\mathrm{J}} \cdot \underline{\lambda} \cdot \overrightarrow{\mathrm{M}},
$$

where $\bar{M}$ is the magnetic moment per rare-earth ion and $\lambda$ is the molecular-field tensor containing the two parameters $\lambda_{1}\left(=\lambda_{x x}=\lambda_{y y}\right)$ and $\lambda_{11}\left(=\lambda_{x z}\right)$.

The calculation of the zero-field susceptibility, magnetic moment, and basal plane anisotropy using the Hamiltonians (1), (5), (7), (8) is described in Refs. 2 and 3.

For the concentrated rare-earth metals and alloys the exchange Hamiltonian is assumed to be of the isotropic Heisenberg form ${ }^{18}$ :

$$
\mathfrak{H}_{\mathrm{ex}}=-\sum_{m \neq n} \mathcal{F}\left(\overrightarrow{\mathrm{R}}_{m}-\overrightarrow{\mathrm{R}}_{n}\right) \overrightarrow{\mathrm{S}}_{m} \cdot \overrightarrow{\mathrm{S}}_{n}
$$

Using (5), (7), and (9), the following formulas for the paramagnetic susceptibilities of a disordered binary alloy are derived in the molecular-field approximation:

$$
1 / \chi_{a, b, c}=1 / \chi_{1 \mathrm{so}}+\Delta_{a, b, c},
$$

where 


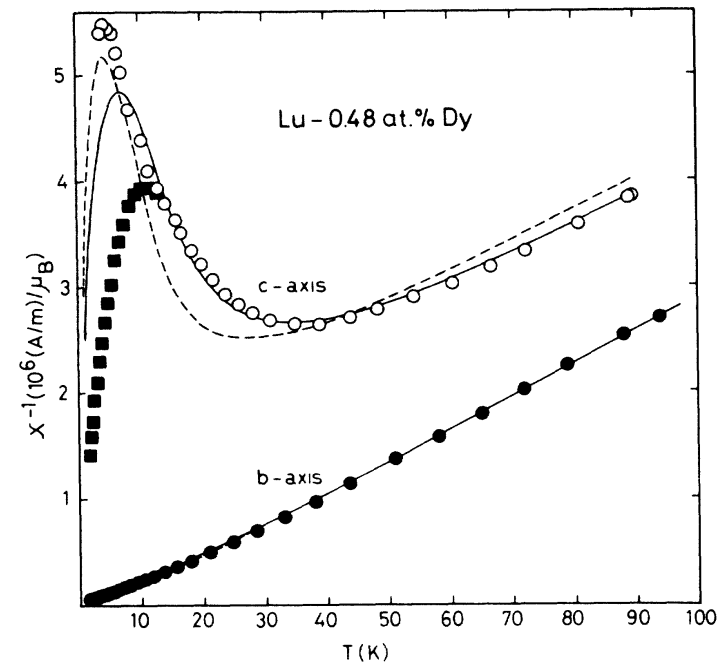

FIG. 1. Reciprocal susceptibilities for Lu-0.48-at.\% Dy. Data used for determining the parameters: $\bullet, \bigcirc$. Uncorrected experimental data (from Ref. 4): 1 . The solid curves are calculated using the parameters in Table I. The dashed curves are calculated using the average crystal-field parameters in Table $\Pi$ and the exchange parameters $\gamma_{\perp}=0$ and $\gamma_{\|}=-0.5 \times 10^{6}(\mathrm{~A} / \mathrm{m}) / \mu_{B}$.

$$
\begin{aligned}
& \frac{1}{\chi_{\text {iso }}}=\frac{3 k}{\mu_{B}^{2}\left(c_{1} g_{1}^{2} \gamma_{1}+c_{2} g_{2}^{2} \gamma_{2}\right)} \\
& \quad \times\left[T-\frac{2 \partial(0)}{3 k}\left(c_{1} \gamma_{1}\left(g_{1}-1\right)^{2}+c_{2} \gamma_{2}\left(g_{2}-1\right)^{2}\right.\right. \\
& \left.\left.\quad-\frac{c_{1} c_{2} \gamma_{1} \gamma_{2}\left(g_{1}-g_{2}\right)^{2}}{c_{1} g_{1}^{2} \gamma_{1}+c_{2} g_{2}^{2} \gamma_{2}}\right)\right], \quad(10) \\
& \Delta_{c}=\left[3 / 5 \mu_{B}^{2}\left(c_{1} g_{1}^{2} \gamma_{1}+c_{2} g_{2}^{2} \gamma_{2}\right)\right] \\
& \quad \times\left[c_{1} g_{1}^{2} \gamma_{1}\left(4 \gamma_{1}-3\right) B_{20}^{(1)}+c_{2} g_{2}^{2} \gamma_{2}\left(4 \gamma_{2}-3\right) B_{20}^{(2)}\right],
\end{aligned}
$$

and $\Delta_{a, b}=-\frac{1}{2} \Delta_{c}$. Terms of order $(1 / T)^{2}$ and higher are neglected. Indexes 1 and 2 refer to the two components, $c$ is the concentration, $\gamma=J(J+1)$, $\mathcal{J}(0)=\sum_{n} \mathcal{F}\left(\bar{R}_{n}\right)$, and $k$ is Boltzmann's constant.

\section{RESULTS FOR DILUTE ALLOYS}

An example of susceptibility data used for the extraction of crystal-field and exchange parameters is shown for Lu-Dy in Fig. 1. The susceptibilities in the $c$ direction at low temperatures have been determined from isothermal magnetization curves as illustrated in Fig. 2. The dashed lines are drawn through points at higher fields for which the theoretical susceptibility is approximately constant (as can be verified from the deduced parameters). The slopes of the dashed lines are expected to be the correct paramagnetic susceptibility. ${ }^{6}$ The susceptibilities determined in this way deviate drastically from the initial susceptibilities as shown in Fig. 1. These deviations, which are attributed to ordering phenomena, are found to be large for all Dy alloys, smaller for $\mathrm{Tb}$ and Ho alloys and negligible for $\mathrm{Er}$ and $\mathrm{Tm}$ alloys. ${ }^{19}$ The corrected susceptibilities are analyzed using the Hamiltonians (1), (5), (7), and (8). In the general case the parameters to be adjusted were $B_{20}, B_{40}, B_{60}, B_{66}, \lambda_{11}, \lambda_{1}$, and the effective concentration $c$ of the rare-earth solute. The number of parameters could however be reduced and unique crystal-field parameters could be found for all the alloy systems. $\lambda_{11}$ and $\lambda_{\perp}$ turned out to be so small that they could be set equal to zero without changing the crystal-field parameters by an amount greater than the final quoted uncertainties. This is valid except for $\mathrm{Y}-\mathrm{Tb}$ and $\mathrm{Lu}-\mathrm{Tb}$ for which $\lambda_{\perp}$ could be set equal to zero but $\lambda_{11}$ had a significant nonzero value. The concentration and for most of the alloys, also $B_{20}$ could be determined independently from the high-temperature data. The concentration could be determined from the slope of the $1 / \chi$ curves and $B_{20}$ from the difference $1 / \chi_{c}-1 / \chi_{b}$.

For alloys with Dy, Ho, or Er all crystal-field parameters could be deduced. Within the accuracy of its determination the ratio $B_{66} / B_{60}$ was the same for these nine alloy systems. The mean value $\left\langle B_{66} / B_{60}\right\rangle=9.7 \pm 1.1$ is very close to the value

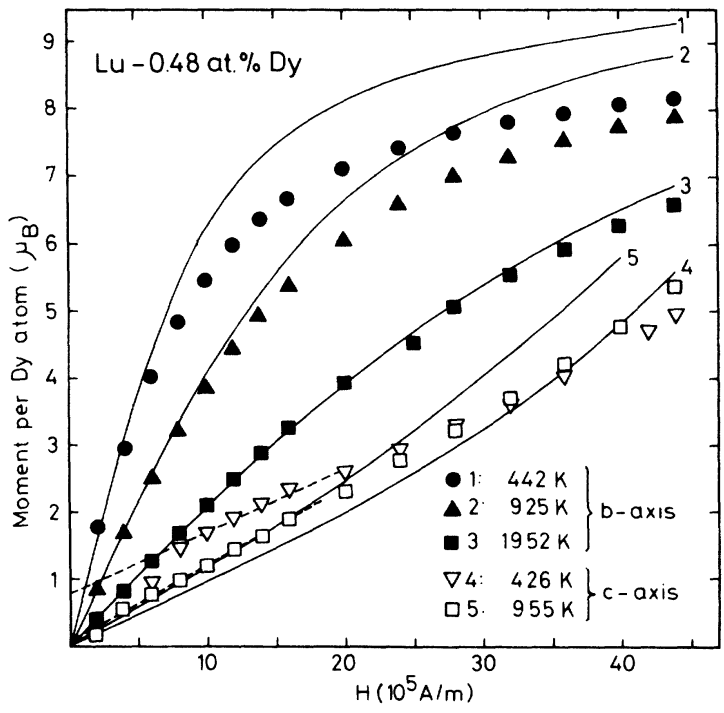

FIG. 2. Magnetization vs field for $\mathrm{Lu}-0.48-$ at. $\% \mathrm{Dy}$ (experimental data from Ref. 4). The slope of the dashed lines gives the paramagnetic susceptibility. Below 4.2 $\mathrm{K}$ this susceptibility cannot be obtained with reasonable accuracy (accuracy $\pm 6 \%$ at $4.2 \mathrm{~K}$ ). The full curves are calculated using the average crystal-field parameters in Table II and the exchange parameters $\gamma_{\perp}=0$ and $\gamma_{11}=-0.5$ $\times 10^{6}(\mathrm{~A} / \mathrm{m}) / \mu_{B}$. 
TABLE I. Values of fitted parameters. $B_{20}, B_{40}$, and $B_{60}$ are deduced for fixed ratio $B_{66} / B_{60}=\frac{77}{8}$. A simultaneous fit of $B_{20}, B_{40}, B_{60}$, and $B_{66} / B_{60}$ for alloys with Dy, Ho, or Er gives approximately the same values of $B_{20}, B_{40}$, and $B_{60}$ and the $B_{66} / B_{60}$ values given in the table. The accuracy of the latter is approximately $15 \%$ for all the alloys. The meansquare deviation $S$ is calculated excluding data below $T_{0}=6 \mathrm{~K} . \quad\left[T_{0}=12 \mathrm{~K}\right.$ in the (hard) $b$ direction for Y-Tm and in the (hard) $c$ direction for Sc-Tm. The data below $12 \mathrm{~K}$ for these alloys and directions could not be satisfactorily fitted, probably due to the high concentrations used.]

\begin{tabular}{|c|c|c|c|c|c|c|c|c|c|}
\hline Alloy & $c \underset{(\%)}{c}$ & $\begin{array}{l}c \text { fit } \\
(\%)\end{array}$ & $B_{20} / \alpha \quad(\mathbf{K})$ & $B_{40} / \beta(\mathrm{K})$ & $B_{60} / \gamma(\mathrm{K})$ & $B_{66} / B_{60}$ & $\gamma_{11}\left(10^{6} \frac{\mathrm{A} / \mathrm{m}}{\mu_{B} / \text { atom }}\right)$ & $\gamma_{\perp}\left(10^{6} \frac{\mathrm{A} / \mathrm{m}}{\mu_{B} / \text { atom }}\right)$ & $S^{1 / 2}(\%)$ \\
\hline \multicolumn{10}{|l|}{$\mathrm{Y}$ host } \\
\hline $\mathrm{Tb}$ & 0.148 & 0.167 & $-88 \pm 15$ & $4.5 \pm 2.5$ & $11.8_{+4}^{-2}$ & $\cdots$ & $-0.7 \pm 0.3$ & 0 & 0.43 \\
\hline Dy & 0.134 & 0.137 & $-115 \pm 12$ & $7.55 \pm 3.5$ & $13.1 \pm 3$ & 8.2 & $-0.3 \pm 0.1$ & 0 & 1.9 \\
\hline Ho & 0.923 & 0.997 & $-91.6 \pm 10$ & $7.4 \pm 2$ & $11.6 \pm 2.5$ & 10.3 & 0 & 0 & 1.8 \\
\hline \multirow[t]{2}{*}{ Er } & 0.142 & 0.142 & $-111 \pm 13$ & $13.5 \pm 6$ & $12.0 \pm 3$ & 11.1 & 0 & 0 & 2.0 \\
\hline & 0.307 & 0.307 & $-111 \pm 10$ & $13.0 \pm 0$ & $12.0 \pm 3$ & 11.1 & 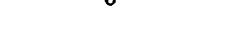 & ( & 2.3 \\
\hline $\mathrm{Tm}$ & 1.75 & 1.61 & $-112 \pm 15$ & $13.0 \pm 6.5$ & $15.1 \pm 7.5$ & $\cdots$ & 0 & 0 & 3.8 \\
\hline \multicolumn{10}{|c|}{ Lu host } \\
\hline $\mathrm{Tb}$ & 0.544 & 0.649 & $-52 \pm 8$ & $4.1 \pm 4$ & $9.5_{-4.5}^{+12}$ & $\cdots$ & $-0.7 \pm 0.15$ & 0 & 1.0 \\
\hline Dy & $(0.557)$ & 0.476 & $-57.1 \pm 7$ & $13.6_{-8}^{+4}$ & $21.8_{-5}^{+3}$ & 9.0 & $-0.2 \pm 0.1$ & 0 & 2.1 \\
\hline Ho & 0.950 & 0.989 & $-46.5 \pm 5$ & $6.3 \pm 3$ & $12.9 \pm 1$ & 9.6 & 0 & 0 & 1.4 \\
\hline $\mathrm{Er}$ & 0.553 & 0.553 & $-55.7 \pm 7$ & $9.6 \pm 2$ & $16.1 \pm 2$ & 10.4 & 0 & 0 & 2.0 \\
\hline $\mathrm{Tm}$ & $\cdots$ & 1.020 & $-56.8 \pm 6$ & $3.4 \pm 2$ & $17.9 \pm 4$ & $\cdots$ & 0 & $-0.15 \pm 0.15$ & 1.6 \\
\hline \multicolumn{10}{|c|}{ Sc host } \\
\hline $\mathrm{Tb}$ & 0.510 & 0.561 & $-31.0 \pm 4$ & $13.4 \pm 5$ & $24.4_{-5}^{+2}$ & $\cdots$ & $-0.1 \pm 0.1$ & 0 & 0.9 \\
\hline Dy & 0.530 & 0.562 & $-29.9 \pm 3$ & $14.6 \pm 4$ & $20.9 \pm 3$ & 9.6 & 0 & 0 & 2.1 \\
\hline Ho & 1.025 & 1.025 & $-20 \pm 8$ & $11.7 \pm 4$ & $14.8 \pm 4$ & 8.5 & 0 & 0 & 2.7 \\
\hline $\mathrm{Er}$ & 0.520 & 0.520 & $-29 \pm 3$ & $8.2_{-2}^{+5}$ & $18.1 \pm 2$ & 10.2 & 0 & 0 & 2.3 \\
\hline $\mathrm{Tm}$ & 1.96 & 1.96 & $-30 \pm 4$ & $4.8 \pm 3.5$ & $23.4 \pm 4$ & $\cdots$ & 0 & 0 & 3.6 \\
\hline
\end{tabular}

$\frac{77}{8}$ given for ideal $c / a$ ratio in the rather general superposition model..$^{20}$ Owing to this fact this ratio was kept fixed in the analysis of susceptibility data for alloys with $\mathrm{Tb}$ and $\mathrm{Tm}$ for which a further reduction of parameters was necessary.

The normalized mean-square deviation

$$
S=\left\langle\left(1 / \chi_{\text {theor }}-1 / \chi_{\text {expt }}\right)^{2}\right\rangle /\left\langle\left(1 / \chi_{\text {theor }}\right)^{2}\right\rangle
$$

was used as a fitting criterion. The terms in $S$ were weighted according to an error in $1 / \chi$ proportional to $1 / \chi$.

In Table I the results for all the alloys used for determining crystal-field parameters are summarized. The errors in the parameters have been estimated from their variation when (a) data below a cutoff temperature $T_{0}$ were excluded with $T_{0}$ varied in the range $4-12 \mathrm{~K}$ (this procedure was adopted to estimate from the analysis the effects of ordering at low temperatures); (b) the concentration and $B_{20}$ were varied within the limits determined from high-temperature data; (c) nonzero molecular-field parameters were included in the fit; (d) $B_{60}$ and $B_{66}$ were fitted independently and with a fixed ratio $B_{66} / B_{60}=\frac{77}{8}$.

Significant enhancements of the concentrations of $\mathrm{Tb}$ alloys and smaller, but still significant, enhancements for some Dy and Ho alloys are observed. These are equivalent to enhancements of the $g$ factor and are believed to be due to conduction electron contributions to the magnetization.

In Figs. 3-5 the crystal-field parameters $B_{l_{m}} / a_{\imath}$ are plotted as a function of rare-earth solute. For a fixed host, $B_{l m} / a_{\imath}$ is approximately constant and no tendency for a variation proportional to $\left\langle r^{l}\right\rangle$, as would be expected from Eqs. (4) and (6) is observed. $\left(\left\langle r^{l}\right\rangle\right.$ decreases from Tb to Tm with a variation compared to the $\mathrm{Tb}$ values of 14, 25, and $36 \%$ for $\left\langle r^{2}\right\rangle,\left\langle r^{4}\right\rangle$, and $\left\langle r^{6}\right\rangle$, respectively. $\left.{ }^{21}\right)$ The study of dilute alloys with light-rare-earth so-

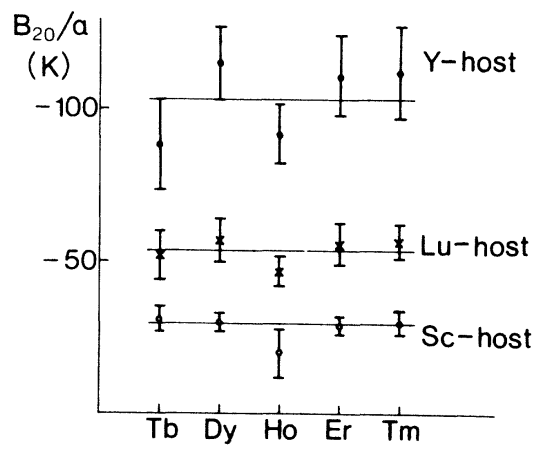

FIG. 3. Crystal-field parameters $B_{20} / \alpha$ plotted for each host as a function of rare-earth solute. The horizontal lines represent mean values for each host. 

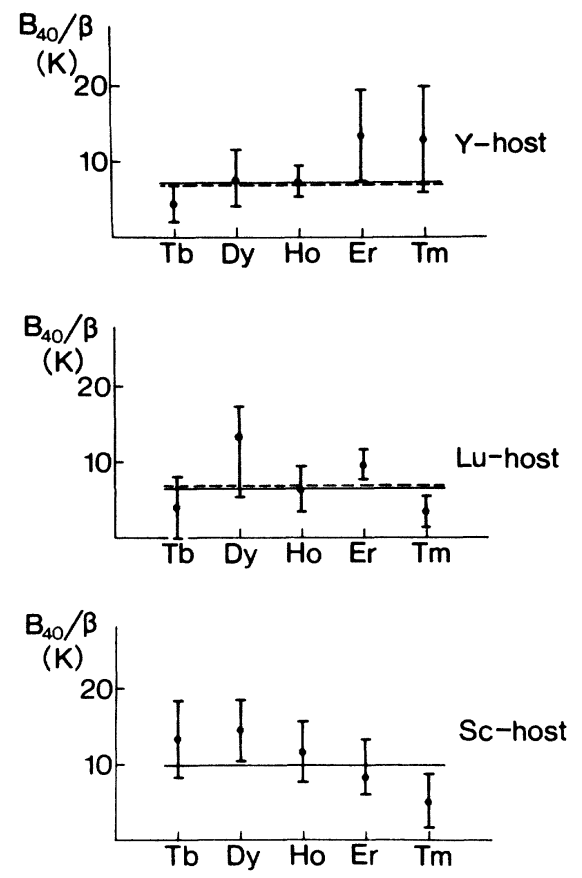

FIG. 4. Crystal-field parameters $B_{40} / \beta$ plotted for each host as a function of rare-earth solute. The horizontal full lines represent mean values for each host. The horizontal dashed lines represent the mean value for both $\mathrm{Y}$ and $\mathrm{Lu}$ hosts.

lutes, ${ }^{22}$ which have much larger mean radii, also indicate that the $B_{\imath m} / a_{l}$ are, to a good approximation, independent of $\left\langle r^{l}\right\rangle$. In Table II mean values and standard deviations are calculated for the parameters for each host. A considerable variation of $B_{20} / \alpha$ for different hosts is observed while $B_{40} /$ $\beta, B_{60} / \gamma$, and $B_{66} / \gamma$ are approximately the same in $\mathrm{Y}$ and $\mathrm{Lu}$ and slightly larger in Sc.

The parameters in Tables I and II were compared to a variety of different experiments. No significant difference of higher-order parameters from the average values for a particular host were detected for any alloy. As shown in Fig. $3, B_{20} / \alpha$ for Ho solutes is significantly smaller than the average value. Variations of parameters with solute may therefore occur and the uncertainties in Table II may be increased to cover the results in Figs. 3-5.

First the parameters in Table II were compared to the susceptibility data for the alloys in Table I and for alloys with varying concentrations. ${ }^{3,19}$ The largest deviations were observed for all alloys with Ho and for Lu-Dy, Fig. 1. These deviations stem from the difference between the average values and fitted values of $B_{20} / \alpha$ for the alloys with Ho, Fig. 3, and of $B_{60} / \gamma$ and $B_{66} / \gamma$ for Lu-Dy, Fig. 5. The latter differences may not be significant. The average parameters reproduce the accurate $b$ -
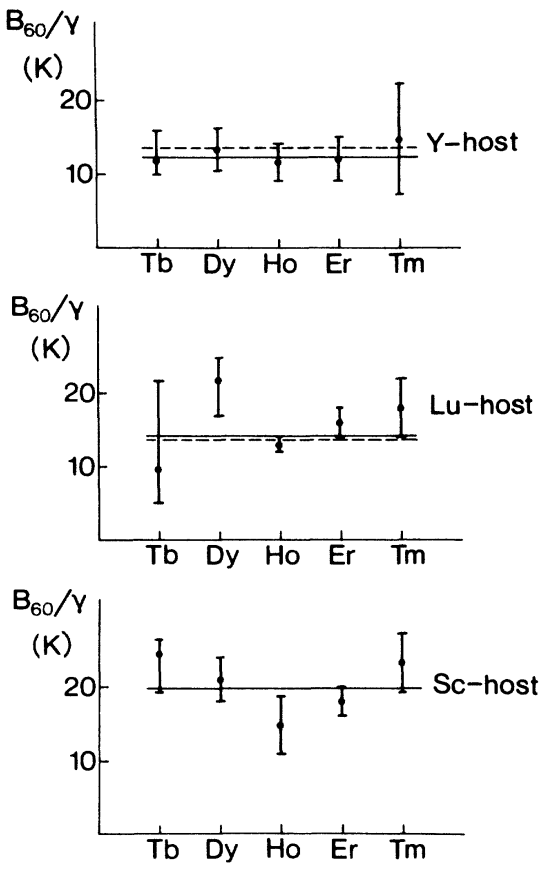

FIG. 5. Crystal-field parameter $B_{60} / \gamma$ plotted for each host as a function of rare-earth solute. Within the accuracy of its determination the crystal-field parameter $B_{66}$ equals $\frac{77}{8} B_{60}$, as described in the text. The horizontal full lines represent the mean values for each host. The horizontal dashed lines represent the mean values for both $\mathrm{Y}$ and $\mathrm{Lu}$ hosts.

axis susceptibilities and the low-temperature features in the $c$-axis susceptibilities better than the fitted values even though the mean-square deviation $S$ is somewhat larger.

Magnetization and basal plane anisotropy measurements are well described by the average parameters except for $\mathrm{Tb}$ and Dy alloys at low temperatures. Generally there are only small differences between earlier published parameters

TABLE II. Average crystal-field parameters for rare earth diluted in $\mathrm{Sc}, \mathrm{Y}$, and $\mathrm{Lu}$. Within the uncertainty of its determination the parameter $B_{66}$ equals $\frac{77}{8} B_{60}$. The inaccuracies are standard mean deviations.

\begin{tabular}{cccc}
\hline \hline Metal & $B_{20} / \alpha(\mathrm{K})$ & $B_{40} / \beta(\mathrm{K})$ & $B_{60} / \gamma(\mathrm{K})$ \\
\hline $\mathrm{Y}$ & $-102.7 \pm 5.7$ & $7.1 \pm 1.2$ & $12.2 \pm 0.4$ \\
$\mathrm{Lu}$ & $-53.4 \pm 2.3$ & $6.5 \pm 1.6$ & $14.1 \pm 1.2$ \\
$\mathrm{Sc}$ & $-29.5 \pm 0.9$ & $9.9 \pm 1.9$ & $19.8 \pm 1.5$ \\
\hline \hline
\end{tabular}




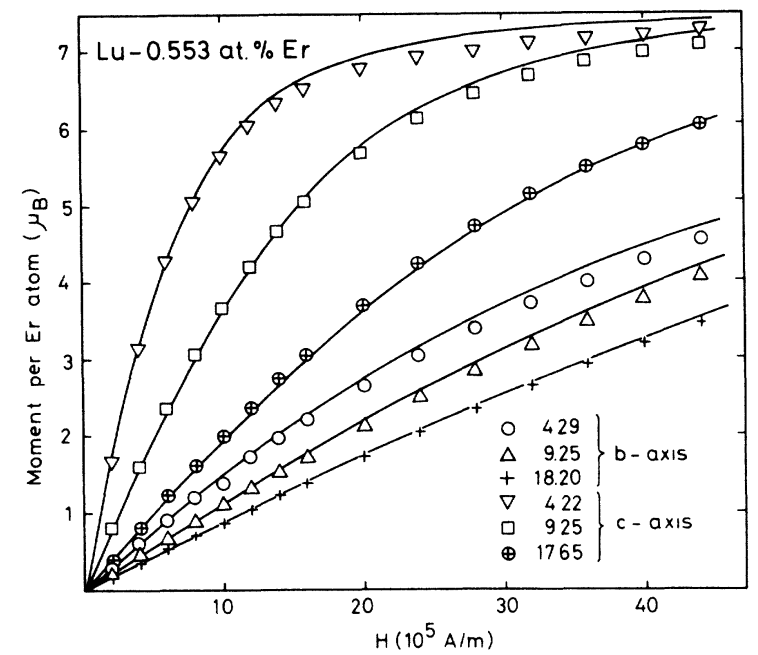

FIG. 6. Magnetization vs field for Lu-0.553-at.\% Er (experimental data from Ref. 4). The curves are calculated using the average crystal-field parameters in Table II neglecting exchange.

and the average parameters deduced here, and therefore the theoretical magnetization and basal plane anisotropy curves in Refs. 2-7 are only slightly modified. For Lu-Dy, ${ }^{4}$ and Y-Dy, ${ }^{2,3}$ the corrections to the susceptibilities have caused large changes in the parameters and data for these alloys will therefore be given as examples here.

The deviations at low temperatures in the magnetization of Lu-Dy, Fig. 2, are characteristic

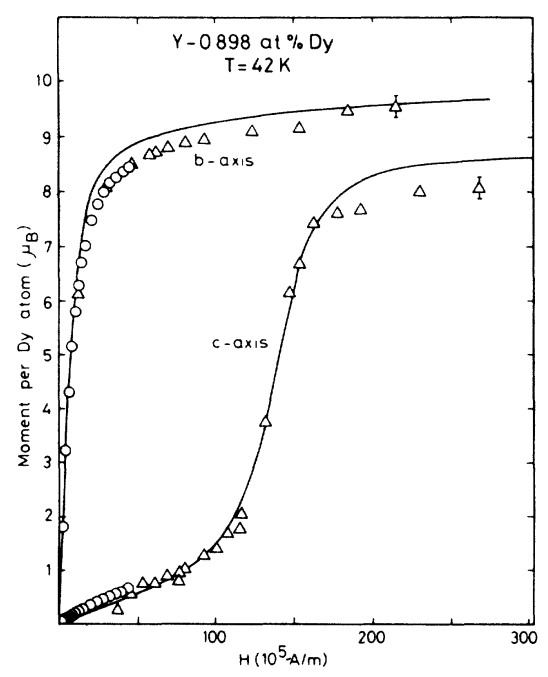

FIG. 7. Magnetization vs field for $\mathrm{Y}-0.898-\mathrm{at} . \%$ Dy (experimental data from Ref. 7). The solid curves are calculated using the average crystal-field parameters in Table II and the exchange parameters in Table I (which are independent of concentration up to $1 \%$ ). for Dy solutes. Alloys with less tendency for ordering show only small deviations even at low temperature as illustrated for Lu-Er in Fig. 6 . Magnetization measurements on $\mathrm{Y}$-Dy up to high fields are shown in Fig. 7 compared with the theoretical curves. For increasing fields in the $c$ direction an approximate $\left|J, J_{z}\right\rangle=\left|\frac{15}{2},-\frac{13}{2}\right\rangle$ state is lowered from about $80 \mathrm{~K}$ above the ground state. In the hexagonal crystal field this state is mixing with the ground state changing this from mainly $\left|J, J_{z}\right\rangle=\left|\frac{15}{2},-\frac{1}{2}\right\rangle$ to mainly $\left|\frac{15}{2},-\frac{13}{2}\right\rangle$ at fields above $150 \times 10^{5} \mathrm{~A} / \mathrm{m}$. This accounts for the abrupt increase in the $c$-axis magnetization.

The basal plane anisotropy, which is found to be very sensitive to ordering, is well described at higher temperatures for all alloys. Large deviations between experimental and theoretical data are however observed at lower temperatures for $\mathrm{Tb}$ and Dy alloys as illustrated in Fig. 8 for Y0.141 -at. \% Dy.

Finally the validity of the average crystal-field parameters are supported by neutron spectroscopy experiments..$^{6,8}$ All neutron transitions predicted theoretically for the alloys studied with neutrons have been observed experimentally as shown in Table III.

\section{RESULTS FOR PURE METALS AND CONCENTRATED ALLOYS}

The study of dilute alloys, supplemented with a study of the pure metals $\mathrm{Tb}$ to $\mathrm{Tm}$ in the paramagnetic phase, allows a reliable estimate of the crystal-field parameters in these magnetic metals. The higher-order parameters for rare-earth solutes in $\mathrm{Y}$ and Lu hosts are approximately the same. Mean values and standard deviations of these parameters for all $\mathrm{Y}$ and Lu alloys are $B_{40} /$

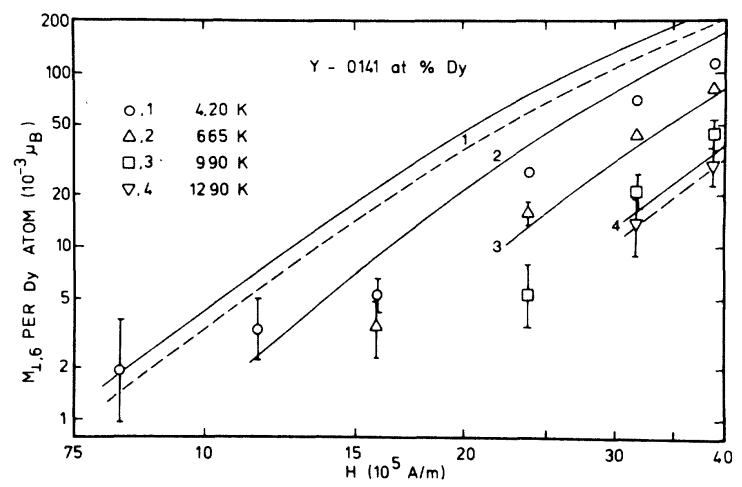

FIG. 8. Perpendicular basal plane sixth-order component vs field and temperature for $\mathrm{Y}-0.141-$ at. \% Dy. The full curves are calculated using the average parameters in Table II and $\lambda_{\perp}=0$. The dashed curves illustrate the effect of a $20 \%$ decrease of $B_{66}$. 
TABLE III. Transitions from the ground state to higher lying states observed in neutron spectroscopy experiments (Refs. 6 and 8). The theoretical values are calculated using the average parameters and the standard mean deviations in Table II. The theoretical values in parentheses are calculated using the fitted parameters in Table I.

\begin{tabular}{|c|c|c|}
\hline Alloy & $\begin{array}{l}\text { Neutron transitions } \\
\text { predicted theoretically }(\mathrm{K})\end{array}$ & $\begin{array}{l}\text { Neutron transitions } \\
\text { observed experimentally (K) }\end{array}$ \\
\hline Y-Dy & $\begin{array}{c}13.3 \pm 0.4 \\
(14.4 \pm 1.9)\end{array}$ & $15.4 \pm 2.3$ \\
\hline \multirow[t]{3}{*}{$\mathrm{Y}-\mathrm{Er}$} & $\begin{array}{l}27.4 \pm 0.6 \\
(26.9 \pm 4.4)\end{array}$ & $26.7 \pm 1.2$ \\
\hline & $\begin{array}{c}49.3 \pm 0.9 \\
(51.9 \pm 6.6)\end{array}$ & $46.4 \pm 2.3$ \\
\hline & $\begin{array}{c}64.4 \pm 2.2 \\
(67.6 \pm 16.9)\end{array}$ & $60.3 \pm 6$ \\
\hline \multirow[t]{2}{*}{ Lu-Er } & $\begin{array}{l}25.1 \pm 1.9 \\
(28.0 \pm 3.0)\end{array}$ & $24.8 \pm 0.5$ \\
\hline & $\begin{array}{l}43.3 \pm 3.0 \\
(48.7 \pm 4.5)\end{array}$ & $46.4 \pm 6.2$ \\
\hline \multirow[t]{2}{*}{$\mathrm{Sc}-\mathrm{Er}$} & $\begin{array}{l}32.2 \pm 2.5 \\
(29.7 \pm 3.2)\end{array}$ & $29.7 \pm 0.7$ \\
\hline & $\begin{array}{c}52.3 \pm 3.5 \\
(48.0 \pm 4.5)\end{array}$ & $51.6 \pm 1.0$ \\
\hline
\end{tabular}

$\beta=6.8 \pm 0.9 \mathrm{~K}, B_{60} / \gamma=13.6 \pm 0.7 \mathrm{~K}$, and $B_{66} / \gamma$ $=(9.7 \pm 1.1) B_{60} / \gamma$. These values-with the inaccuracies may be increased to cover values for the specific solute in both $\mathrm{Y}$ and $\mathrm{Lu}-$ are expected to be valid also in the metals $\mathrm{Tb}$ to $\mathrm{Tm}$. This is due to the fact (a) the metals $\mathrm{Tb}$ to $\mathrm{Tm}$ are very similar to $\mathrm{Y}$ and $\mathrm{Lu}$ and have lattice parameters and conduction bandwidths ${ }^{23}$ lying between those for $\mathrm{Y}$ and $\mathrm{Lu}$; (b) $B_{40} / \beta, B_{60} / \gamma$, and $B_{66} / \gamma$ are approximately independent of whether the $4 f$ shell is empty (Y) or filled (Lu); (c) $B_{40} / \beta, B_{60} /$ $\gamma$, and $B_{66} / \gamma$ are insensitive parameters as can be seen by comparison of the value for $\mathrm{Y}$ and $\mathrm{Lu}$ to those for Sc (Sc is significantly different from $Y$ and $\mathrm{Lu}$ both with respect to lattice parameters and conduction band $\mathbf{s}^{23}$ ). Point (c) suggests that these parameters may also be representative for the lightrare-earth metals.

$B_{20} / \alpha$ is a host-sensitive parameter. Simple theories predict a variation of $B_{20}$ with lattice constant with the $c / a$ ratio as the dominant factor. Experimentally an approximately linear variation of $B_{20} / \alpha$ with $c / a$ ratio of the host is observed for $\mathrm{Lu}, \mathrm{Sc}$, and $\mathrm{Y}$ hosts as illustmted in Fir. 9 . Owing to the similarities of $\mathrm{Lu}$ and $\mathrm{Y}$ to the metals $\mathrm{Tb}$ to $\mathrm{Tm}$ preliminary estimates of $B_{20} / \alpha$ in these metals may be obtained from the dashed line in Fig. 9 drawn between the points for $\mathrm{Y}$ and $\mathrm{Lu}$. Deviations between these values of $B_{20} / \alpha$ and the correct values may be expected since $\mathrm{Y}$ is not a rare-earth metal and $B_{20} / \alpha$ is a sensitive parameter.
Correct values of $B_{20} / \alpha$ could however be obtained from measurements of the paramagnetic susceptibility up to high temperatures of the pure rare-earth metals. Typical susceptibility data are shown for Dy in Fig. 10. The reciprocal susceptibilities vary linearly with temperature (except close to $T_{N}$ ) for all the heavy-rare-earth metals. Above $T_{N}$ the difference $\left|1 / \chi_{c}-1 / \chi_{b}\right|$ is a decreasing function of temperature for all metals as illustrated in Fig. 11. (Below $T_{N}$ the tempera-

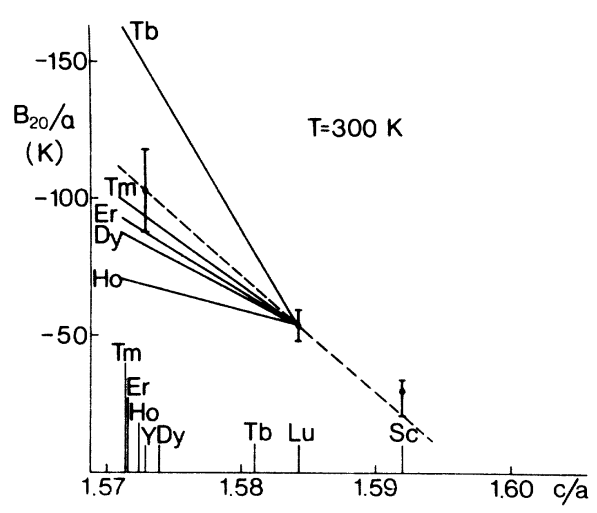

FIG. $9-1$-field parameter $B_{20} / \alpha$ plotted as a function of $c / a$ cicul of the hoss. Points with error bars are average values for rare-earth solutes in $\mathrm{Y}, \mathrm{Lu}$, or Sc uncertainties in Table II have been increased. The dashed line is drawn through the points for $\mathrm{Y}$ and $\mathrm{Lu}$. The full lines are estimates of $B_{20} / \alpha$ for a specific rare-earth ion in Lu or in concentrated heavy-rareearth metals or alloys. 


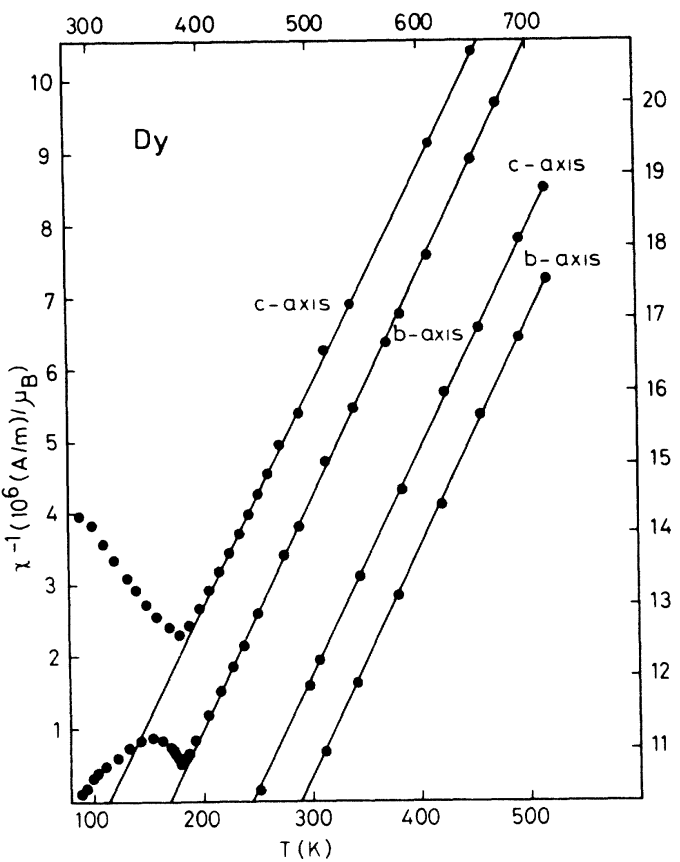

FIG. 10. Reciprocal initial susceptibilities of Dy. The left-hand and bottom scales refer to the two curves on the left-hand side, while the top and right-hand scales refer to the two curves on the right-hand side.

ture dependence of $\left|1 / \chi_{c}-1 / \chi_{b}\right|$ is not simple due to complicated magnetic ordering and magnetostrictive effects. ${ }^{24}$ ) Effective values of $B_{20} / \alpha$ which include possible contributions from anisotropic ex-

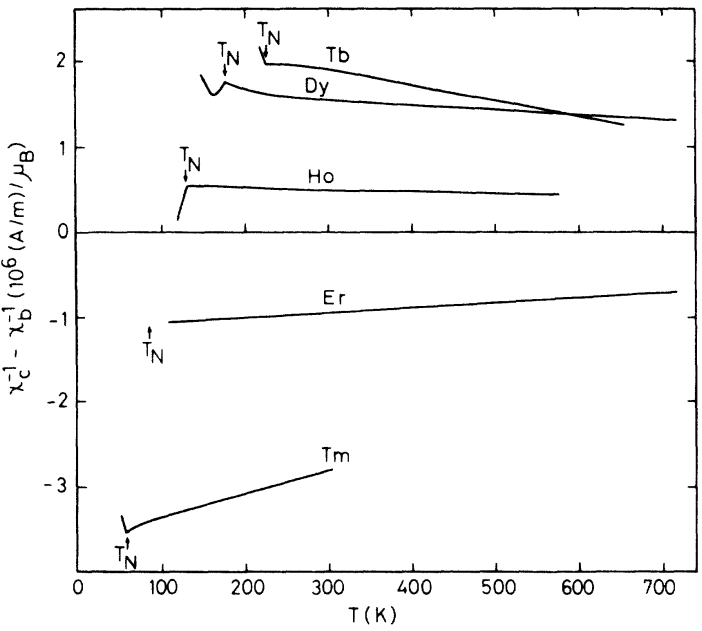

FIG. 11. Difference between reciprocal initial susceptibilities vs temperature for the heavy-rare-earth metals.

change can be derived from the data in Fig. 11. The values $B_{20}^{\text {eff }} / \alpha$ at $300 \mathrm{~K}$ are given in Table IV. Comparison to the preliminary estimates of $B_{20} / \alpha$ indicate that contributions to $1 / \chi_{c}-1 / \chi_{b}$ from anisotropic exchange is rather small (30\% in $\mathrm{Tb}$ and smaller in Dy to $\mathrm{Tm}$ ). The linear variation of $1 / \chi_{c}-1 / \chi_{b}$ in Fig. 11 is interpreted as due to a linear variation of lattice parameters with temperature..$^{25}$ Since the overall changes of $1 / \chi_{c}-1 / \chi_{b}$ are larger than the contributions from anisotropic exchange the temperature coefficient $d B_{20} / d T$ can to

TABLE IV. Crystal-field parameters at $T=300 \mathrm{~K}$ for the pure rare-earth metals. The parameter $B_{66}$ equals $(9.7 \pm 1.1) B_{60} . B_{20}^{\text {eff }}$ is determined from $1 / \chi_{c}-1 / \chi_{b}$ for the pure metals. $d\left(B_{20} /\right.$ $\alpha) / d(c / a)=\left[d\left(B_{20} / \alpha\right) / d T\right] d T / d(c / a) . \quad d\left(B_{20} / \alpha\right) / d T$ is determined from the measurements in Fig. 12 and similar measurements in the temperature range $300-400 \mathrm{~K}$ published in Ref. 26 . (The uncertainties cover results for both types of measurement except for Dy in which effects of higher-order parameters are significant at $300-400 \mathrm{~K}$. The values in the table for Dy are therefore deduced from the data in Fig. 12.) $d T / d(c / a)$ is derived from lattice parameter measurements on $\mathrm{Tb}, \mathrm{Dy}, \mathrm{Ho}$, and $\mathrm{Er}$ in Ref. $25[d T / d(c / a)$ for $\mathrm{Tm}$ is an extrapolated value]. In estimating the inaccuracies for $B_{20} / \alpha$ the individual values of $B_{20} / \alpha$ for rare-earth solutes in Lu have been included. Mean values of $B_{40} / \beta$ and $B_{60} / \gamma$ in Lu and Y calculated for each magnetic rare-earth impurity separately are quoted. The values in parentheses assume no variation of these parameters with rare-earth solute.

\begin{tabular}{|c|c|c|c|c|c|c|}
\hline Metal & $\begin{array}{l}B_{20}^{\text {eff }} / \alpha \\
(\mathrm{K})\end{array}$ & $10^{2} \frac{d\left(B_{20} / \alpha\right)}{d T}$ & $\begin{array}{c}\frac{d\left(B_{20} / \alpha\right)}{d(c / a)} \\
\left(10^{4} \mathrm{~K}\right)\end{array}$ & $\begin{array}{l}B_{20} / \alpha \\
(\mathrm{K})\end{array}$ & $\begin{array}{l}B_{40} / \beta \\
(\mathrm{K})\end{array}$ & $\begin{array}{l}B_{60} / \gamma \\
(\mathrm{K})\end{array}$ \\
\hline $\mathrm{Tb}$ & $-99 \pm 4$ & $6.8 \pm 2.5$ & $0.84 \pm 0.31$ & $-81_{-13}^{+10}$ & $\begin{array}{c}4.4 \pm 2.1 \\
(6.8 \pm 0.9)\end{array}$ & $\begin{array}{c}11.5 \pm 2.8 \\
(13.6 \pm 0.7)\end{array}$ \\
\hline Dy & $-100 \pm 4$ & $4.2 \pm 1.7$ & $0.25 \pm 0.09$ & $-81_{-10}^{+15}$ & $\begin{array}{c}9.1 \pm 3.0 \\
(6.8 \pm 0.9)\end{array}$ & $\begin{array}{c}16.2 \pm 2.4 \\
(13.6 \pm 0.7)\end{array}$ \\
\hline Ho & $-81 \pm 4$ & $2.7 \pm 1.2$ & $0.13 \pm 0.06$ & $-69_{-14}^{+7}$ & $\begin{array}{c}7.1 \pm 1.7 \\
(6.8 \pm 0.9)\end{array}$ & $\begin{array}{c}12.7 \pm 0.9 \\
(13.6 \pm 0.7)\end{array}$ \\
\hline $\mathrm{Er}$ & $-124 \pm 6$ & $7.4 \pm 2.5$ & $0.30 \pm 0.10$ & $-92_{-12}^{+16}$ & $\begin{array}{c}9.9 \pm 1.8 \\
(6.8 \pm 0.9)\end{array}$ & $\begin{array}{c}14.8 \pm 1.7 \\
(13.6 \pm 0.7)\end{array}$ \\
\hline $\mathrm{Tm}$ & $-107 \pm 8$ & $10.2 \pm 2.1$ & $0.35 \pm 0.07$ & $-99_{-9}^{+14}$ & $\begin{array}{c}4.2 \pm 1.9 \\
(6.8 \pm 0.9)\end{array}$ & $\begin{array}{r}17.3 \pm 3.5 \\
(13.6 \pm 0.7)\end{array}$ \\
\hline
\end{tabular}




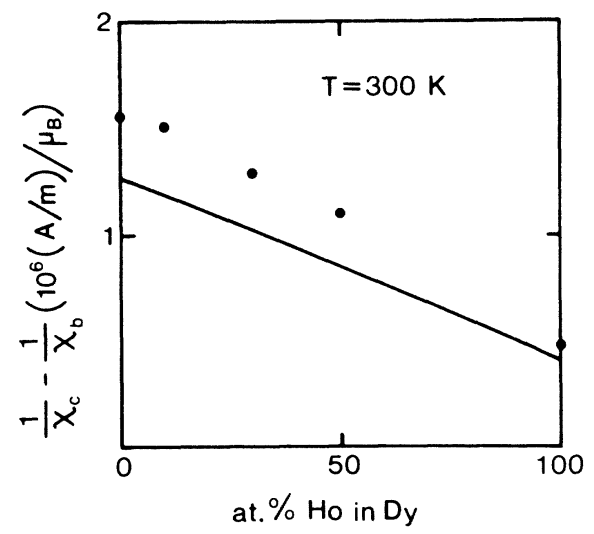

FIG. 12. Difference between reciprocal initial susceptibilities versus concentration for the Dy-Ho alloy system. The solid curve shows the theoretical crystalfield predictions. Contributions from higher-order parameters have been included as a perturbation [these parameters contribute $0.055 \times 10^{6}(\mathrm{~A} / \mathrm{m}) / \mu_{B}$ to $1 / \chi_{c}$ $-1 / \chi_{b}$ at $300 \mathrm{~K}$ in $\left.\mathrm{Dy}\right]$.

a good approximation be determined from Fig. 11. From $d B_{20} / d T$ determined in this way and from published lattice parameters $\mathrm{s}^{25}$ the slope $d\left(B_{20} / \alpha\right) /$ $d(c / a)$ of the variation of $B_{20} / \alpha$ with $c / a$ can be derived for each rare-earth metal. These slopes are expected to be approximately constant within the narrow range of $c / a$ values in Fig. 9 as also indicated by the results in Fig. 11. The rareearth metals $\mathrm{Tb}$ to $\mathrm{Tm}$ are very similar to $\mathrm{Lu}$ and have lattice parameters which deviate less than $3 \%$ from those of Lu. $B_{20} / \alpha$ in Lu is therefore expected to be representative for the parameter in $\mathrm{Tb}$ to $\mathrm{Tm}$ except for differences due to $c / a$ ratio. Curves for $B_{20} / \alpha$ vs $c / a$ for each rare-earth metal can now be drawn as shown in Fig. $9 . B_{20} /$

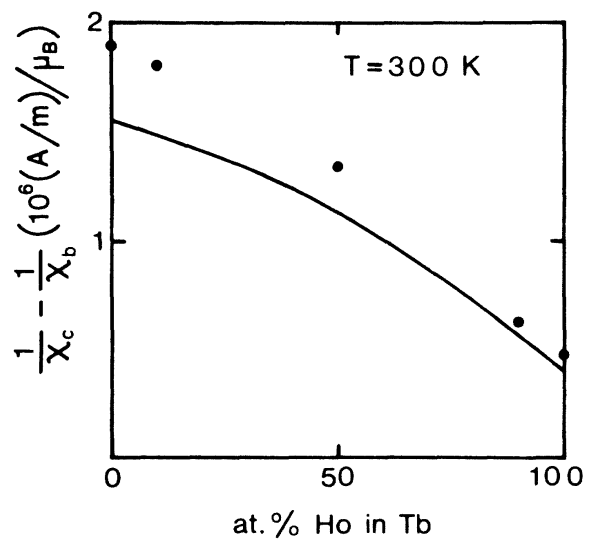

FIG. 13. Difference between reciprocal initial susceptibilities vs concentration for the Tb-Ho alloy system. The solid curve is the theoretical crystal-field prediction. $\alpha$ in the pure rare-earth metals derived from these curves are collected in Table IV. Comparison to $B_{20}^{\text {eff }} / \alpha$ in the table indicates a contribution from anisotropic exchange to this parameter of approximately $20 \%$ for $\mathrm{Tb}$, Dy, Ho, and $\mathrm{Er}$ and may be smaller for $\mathrm{Tm}$.

The crystal-field parameters $B_{20} / \alpha$ can be checked by paramagnetic measurements on inter heavy-rare-earth alloy systems. The $c / a$ ratio is expected to vary linearly across these alloy systems as reported for Ho-Tb. ${ }^{27}$ Using Eq. (10) with $B_{20} / \alpha$ for each component obtained from Fig. 9 , the variation of $1 / \chi_{c}-1 / \chi_{b}$ over the alloy systems can be derived. In Figs. 12-14 experimental and theoretical data are compared for the alloy systems Ho-Dy, Tb-Ho, and Tb-Er. The agreement is good in all cases, taking into account the expected contribution from anisotropic exchange of approximately $20 \%$ of the experimental anisotropy. For the Ho-Dy alloy system the variation of $c / a$ ratio is small resulting in an approximately linear variation of $1 / \chi_{c}-1 / \chi_{b}$ over the alloy system. For the $\mathrm{Tb}-\mathrm{Ho}$ and $\mathrm{Tb}-\mathrm{Er}$ alloy systems the different values of $d\left(B_{20} / \alpha\right) / d(c / a)$ for the two components give rise to the characteristic nonlinear variation, which is also shown by experimental data.

The higher-order parameters in the heavy-rareearth metals and alloys (given in Table IV) cannot be checked with sufficient accuracy by paramagnetic measurements, due to the high ordering temperatures. However the approximately linear variation of $1 / \chi_{c}-1 / \chi_{b}$ in Fig. 11 for Tb, Ho, Er,

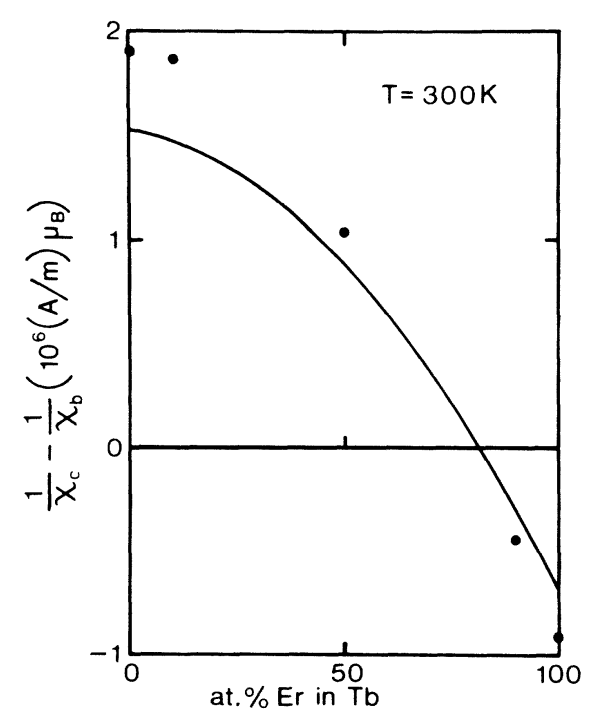

FIG. 14. Difference between reciprocal initial susceptibilities versus concentration for the Tb-Er alloy system. The solid curve is the theoretical crystal-field prediction. 
and $\mathrm{Tm}$ and the small deviations from linearity for Dy are consistent with the theoretical crystal-field predictions.

\section{CONCLUSION}

The research reviewed in the present paper has led to a determination of the crystal-field interactions on the magnetic heavy-rare-earth ions $\mathrm{Tb}$, Dy, Ho, Er, or Tm in the following systems: the unmagnetic hosts Sc, $\mathrm{Y}$, and $\mathrm{Lu}$; the magnetic rare-earth metals $\mathrm{Tb}$, Dy, Ho, Er, and $\mathrm{Tm}$; and inter-heavy-rare-earth alloys.

The deduced crystal-field parameters for the rare-earth ions in Sc, Y, and Lu (Table I) describe consistently the results obtained in many different experiments. These include measurements of the temperature dependence of the lowfield susceptibility and medium field (6 T) magnetization, high-field $(38 \mathrm{~T})$ magnetization measurements at $4.2 \mathrm{~K}$, experiments on the basal plane anisotropy, and inelastic neutron scattering experiments. All of these experiments were performed on many alloy systems. Measurements on alloys of different concentrations revealed no dependence on concentration of the crystal-field parameters. The parameters for rare-earth solutes in a particular host show no significant dependence on solute in the case of $B_{40} / \beta, B_{60} / \gamma$, or $B_{66} / \gamma$. It turns out that the average parameters in Table II are in satisfactory agreement with all the above experiments. Discrepancies between experimental and theoretical results which exist for some alloys at low temperature indicate effects of magnetic ordering. This ordering, the anisotropic molecular-field parameters, and the conduction-electron contributions to the $g$ factor and to the saturation moments (Fig. 2) are of interest for the study of exchange interactions. Further experimental and theoretical investigations of these phenomena would be of interest.

The remarkable insensitivity of the higher-order parameters $B_{40} / \beta, B_{60} / \gamma$, and $B_{66} / \gamma$ to the host indicates that the values of these parameters for a specific magnetic rare-earth ion in $\mathrm{Y}$ and Lu give a reliable estimate of the values in the pure magnetic rare-earth metal (Table IV). Since $B_{40} / \beta$, $B_{60} / \gamma$, and $B_{66} / \gamma$ seem to be the same in all $\mathrm{Y}$ and $\mathrm{Lu}$ alloys, average values quoted in parentheses in Table IV, may give a more accurate estimate of these crystal-field parameters in the pure rare-earth metals. These values are expected to be valid also for inter-heavy-rare-earth alloy systems.

$B_{20} / \alpha$ is sensitive to the host as well as to the solute. $B_{20} / \alpha$ for a specific rare-earth ion in $\mathrm{Tb}$, $\mathrm{Dy}, \mathrm{Ho}, \mathrm{Er}, \mathrm{Tm}$, or Lu or in alloys between these is determined by the $c / a$ ratio of the metal or alloy (Fig. 9). $B_{20} / \alpha$ in these metals and alloys accounts for approximately $80 \%$ of the paramagnetic anisotropy (Table IV and Figs. 12-14). The crystal-field interactions thus account for the characteristic variation of the paramagnetic anisotropy in the heavy-rare-earth alloy systems and across the heavy-rare-earth series (especially the large difference between the anisotropy for Ho and Er).

The crystal-field parameters determined in the present project are of importance for the quantitative understanding of the physical properties of the heavy-rare-earth metals and alloys. Of theoretical interest is the lack of variation of the crystal-field parameters with the $4 f$ radii, $\left\langle r^{l}\right\rangle$, and the characteristic $c / a$ dependence of $B_{20} / \alpha$ for each rare-earth ion. These results, which are not fully consistent with present with present theories of the crystal fields in the rare-earth metals, point to the need for further first-principles calculations.

\section{ACKNOWLEDGMENTS}

The author is grateful to Dr. J. Hфg for his ideal collaboration on the first part of this project. The author is also indebted to Professor V. Frank and Professor A. R. Mackintosh for many fruitful discussions during the course of this research. Dr. P. Fynbo is thanked for discussions and for performing the measurements on Tm. S. Balling and K. M. Ravn participated in the measurements on the Ho-Dy and Tb-Er alloys.
*Present address.

${ }^{1}$ P. Touborg and J. Høg, Phys. Rev. Lett. 33, 775 (1974).

${ }^{2} \mathrm{~J}$. Hळg and P. Touborg, Phys. Rev. B 9, 2920 (1974).

${ }^{3}$ J. Hog and P. Touborg, Phys. Rev. B 11,520 (1975).

${ }^{4}$ P. Touborg and J. Høg, Phys. Rev. B 11,2660 (1975).

${ }^{5} \mathrm{~J}$. Høg and P. Touborg, Phys. Rev. B 14,1209 (1976).

${ }^{6} \mathrm{O}$. Rathmann and P. Touborg, following paper, Phys. Rev. B 16, 1212 (1977).

${ }^{7}$ P. Touborg, J. Høg, G. J. Cock, and L. W. Roeland, Phys. Rev. B 10, 2952 (1974).
${ }^{8}$ O. Rathmann, J. Als-Nielsen, P. Bak, J. H6g, and P. Touborg, Phys. Rev. B 10, 3983 (1974).

${ }^{9} \mathrm{~K}$. A. McEwen and P. Touborg, J. Phys. F $\underline{3}, 1903$ (1973).

${ }^{10}$ P. B. Fynbo, O. V. Nielsen, and P. Touborg, J. Phys. F 8, 631 (1975).

${ }^{11} \mathrm{~T}$. Johansson and K. Gynther Nielsen, J. Phys. E $\underline{9}$, 852 (1976).

${ }^{12}$ L. W. Roeland, F. A. Muller, and R. Gersdorf, Colloq. Int. Cent. Natl. Rech. Sci. 166, 175 (1967). 
${ }^{13}$ O. V. Nielsen and V. I. Zaitzev, J. Phys. E $\underline{6}, 1022$ (1973).

${ }^{14} \mathrm{H}$. B. Moller and M. Nielsen, in Instrumentation for Neutron Inelastic Scattering Research, Proceedings of the panel held in Vienna, 1-5 Dec. 1969, (IAEA, Vienna, 1970), pp. 49-70.

${ }^{15} \mathrm{~S}$. Foner, Rev. Sci. Instrum. 27, 548 (1956).

${ }^{16}$ P. Touborg, J. Phys. (Paris) 32 , Cl-241 (1971).

${ }^{17} \mathrm{~A}$. Abragam and B. Bleaney, Electron Paramagnetic Resonance of Transition Ions (Clarendon, Oxford, 1970).

${ }^{18}$ M. A. Ruderman and C. Kittel, Phys. Rev. 96, 99 (1954); T. Kasuya, Prog. Theor. Phys. 16, 45 (1956); K. Yosida, Phys. Rev. 106, 893 (1957).

${ }^{19}$ The initial susceptibilities of alloys with $\mathrm{Dy}, \mathrm{Tb}$, and Ho published in Refs. 1-4 need corrections at low temperatures in the $c$ direction to give paramagnetic susceptibilities. Corrected data for Sc alloys are given in Ref. 5, for Y-0.14-at.\% Dy in Ref. 6 and for Lu-0.5at. \% Dy in the present paper. For Y-0.17-at.\% Tb, Lu-0.6-at.\% Tb, and $\mathrm{Lu}-1.0-$ at. \% Ho the correction factor to the reciprocal initial susceptibilities goes smoothly from 1 at $12 \mathrm{~K}$ to $1.10,1.17$, and 1.17 , respectively, at $4.2 \mathrm{~K}$. Corrected susceptibilities are independent of concentration up to 1 at. \%.

${ }^{20}$ D. J. Newman, Adv. Phys. 20, 197 (1971).

${ }^{21}$ A. J. Freeman and R. E. Watson, Phys. Rev. 127, 2058 (1962).

${ }^{22} \mathrm{P}$. Touborg, R. Nevald, and T. Johansson (unpublished). ${ }^{23} \mathrm{O}$. Jepsen and $\mathrm{O}$. K. Andersen (private communications) quote the following values for the $d$-conductionband width in units of rydbergs: Sc, $0.28 ; \mathrm{Y}, 0.48$; $\mathrm{Tb}, 0.59$; and $\mathrm{Lu}, 0.61$ (obtained by the standard potential construction using superposition of atomic charge densities and full Slater exchange).

${ }^{24}$ P. B. Fynbo, J. Phys. F (to be published).

${ }^{25} \mathrm{~J}$. Rhyne, in Magnetic Properties of Rare Earth Metals, edited by R. J. Elliott (Plenum, New York, 1972), and references therein.

${ }^{26}$ R. Aleonord, P. Boutron, and D. Bloch, J. Phys. Chem. Solids, 30, 2277 (1969).

${ }^{27}$ F. H. Spedding, R. G. Jordan, and R. W. Williams, J. Chem. Phys. 51, 99 (1954). 